\title{
Tissue Doppler Derived Longitudinal Strain and Strain Rate During the First 3 Days of Life in Healthy Term Neonates
}

\author{
EIRIK NESTAAS, ASBJØRN STØYLEN, LEIF BRUNVAND, AND DRUDE FUGELSETH
}

Department of Pediatrics [E.N., L.B., D.F.], Ullevål University Hospital, 0407 Oslo, Norway; Department of Pediatrics [E.N.], Vestfold Hospital Trust, 3103 Tonsberg, Norway; Department of Cardiology [A.S.], St. Olavs Hospital, 7006 Trondheim, Norway; Department of Circulation and Medical Imaging [A.S.], Norwegian University of Science and Technology, 7489 Trondheim, Norway; Faculty of Medicine [D.F.], University of Oslo, 0318 Oslo, Norway

\begin{abstract}
Tissue Doppler derived longitudinal strain and strain rate were studied in 48 healthy term neonates by measuring peak systolic strain (PSS) and peak systolic strain rate (PSSR) in 18 heart segments on day 1-3 of life. The mean PSS for each examination was $-21.8 \%(-22.1,-21.4)$ [mean $(95 \% \mathrm{CI})$ ], and the mean PSSR was $-1.78 / \mathrm{s}(-1.81,-1.74)$. Age $(\mathrm{d})$, fetal shunts, and heart rate had negligible impact on measurements for any segment. The fractional shortening had negligible impact on septum and left heart segments and the pulmonary artery pressure had no impact on the right heart segments. Values varied significantly between segments and individuals, and were lower (closer to zero) in images of low quality than in images of high quality $(p<0.05)$. Both apical and basal segments values were lower in the septum and higher in the right ventricle than in the left ventricle $(p<0.05)$, except for no difference between the basal left and right segments PSSR. Apical segments values were higher than basal segments values in the right ventricle $(p<0.05)$ but not in the septum or the left ventricle. At present, PSS and PSSR are more feasible for quantifying differences between segment groups and patient groups than between individuals and segments within individuals. (Pediatr Res 65: 357-362, 2009)
\end{abstract}

$\mathrm{S}^{\mathrm{tn}}$ rain and strain rate assess function in heart segments (1). Strain describes the relative change in heart segment length and strain rate is strain per time unit. Data on strain and strain rate measurements in neonates are scarce. We have previously found strain and strain rate by tissue Doppler feasible and reliable in neonates (2). Little is known about changes in myocardial function during the first days of life, when the hemodynamic circulatory system changes from two parallel to one serial circuit, the gas exchange is transferred from the placenta to the lungs and the fetal shunts are gradually closed (3). Strain and strain rate measurements could provide new knowledge about the myocardial function during these changes, and also serve as a reference for studies of the heart function in pathologic states during this period.

The aim of the present study was to investigate myocardial contraction in healthy term neonates by measuring longitudinal peak systolic strain (PSS) and strain rate (PSSR) in the first 3 days of life and assess the impact of age, fetal shunts, image quality, and different heart regions.

Received June 9, 2008; accepted September 23, 2008.

Correspondence: Eirik Nestaas, M.D., Department of Pediatrics, Vestfold Hospital Trust, 3103 Tønsberg, Norway; e-mail: eirikpda@start.no

Supported by the Eastern Norway Regional Health Authority, the Southern Norway Regional Health Authority and Renée og Bredo Grimsgaard's Foundation.

\section{METHODS}

Subjects. Forty-eight healthy term neonates from the maternity ward at Ullevål University Hospital were examined on the first, second, and third day of life (March-May 2005) (Table 1). One neonate had an apical ventricular septum defect. The measurements from this neonate were not excluded because the defect was without hemodynamic significance and lied outside the myocardial region used in the analyses. All other neonates had structurally normal hearts.

The project was approved by the Regional Committee for Medical Research Ethics and by the scientific committee at Ullevål University Hospital. Written informed parental consent was obtained.

Measurements. To minimize noise in the analyses, a $2.4 \mathrm{MHz}$ phase array probe (5S probe, Vivid 7 Dimension v 4.0.1 built 1644, GE Vingmed, Horten, Norway) was used (4). Apical tissue Doppler images were recorded with the wall parallel to the ultrasound beams using tissue velocity range $-16-16$ $\mathrm{cm} / \mathrm{s}$ and default settings for frame rate and for lateral (step 0 of 4 ) and radial (step 3 of 3) averaging. Images for assessment of fractional shortening and fetal shunts were recorded at each examination.

Protocol. A model for longitudinal strain and strain rate analyses of six left ventricle and septum walls has been suggested in adults (5). Right ventricle models have not been suggested. Two right ventricle walls have been studied in children (6). The right superior wall to our knowledge has not been assessed by ultrasound earlier, but contraction in these segments has been assessed by MRI $(7,8)$ in adults. Three segments (basal, mid, and apical) are usually studied in each wall. The three-segment model is basically related to the coronary circulation, which is less relevant in neonates. The noise-tosignal ratio is high, especially in short segments $(1,9)$. As the neonatal heart is much smaller than the adult heart (ventricle length $3 \mathrm{~cm}$ versus $10 \mathrm{~cm}$ ), segments will be very short in a three-segment model. This might lead to a signal-to-noise ratio too inferior for analysis, and to increase the segment length a two-segment model (basal and apical) was used (Fig. 1).

Five apical views were used to study nine walls; the six walls usually studied in adults (5), the two right ventricle walls previously studied in children (6) and the right superior wall. The left lateral wall, the septum and the right lateral wall were studied from the four-chamber view, the left inferior and left anterior wall from the left two-chamber view, the left inferiolateral wall and the anterior septum from the apical long-axis view, the right inferior wall from the right inferior two-chamber view and the right superior wall from the right superior two-chamber view. The two latter views were achieved by tilting the probe from the four-chamber view to get the right ventricle in center and then rotate the probe counter clockwise for the right inferior two-chamber view and clockwise for the right superior two-chamber view.

Data analysis. Analyses were done in the EchoPac PC analysis software (GE Vingmed, Horten, Norway). In most images, the sector angel was approximately $30^{\circ}$ and the tissue Doppler frame rate 170-220/s. The PSS and PSSR were extracted from strain and strain rate curves averaged for three consecutive cycles (Fig. 2). To minimize noise in the analysis, a region of interest length $1 \mathrm{~mm}$, region of interest width $3 \mathrm{~mm}$, strain length $10 \mathrm{~mm}$, and $40 \mathrm{~ms}$ Gaussian smoothing was used (2). Linear drift compensation was used for the Lagrangian strain curves. A semi-automatic tracking algorithm kept the region of interest centered in the segment during the cardiac cycle. End diastole was defined by the ECG R-peak and end systole by the notch in the displacement curve caused by the closure of the aortic and pulmonary valves (10). The image quality $(1-4 ; 1=$ good, 4 = inferior for analysis) and ultrasound beam alignment with the segment longitudinal direction $(1-3 ; 1=\operatorname{good}, 3=$ inferior for analysis) were assessed

Abbreviations: PSS, peak systolic strain; PSSR, peak systolic strain rate 
Table 1. Data for the neonates examined

$\begin{array}{lc}\text { No of neonates (female:male) } & 48(1: 1) \\ \text { Gestational age (mean and range) (wks) } & 41(37-42) \\ \text { Birth weight [mean (SD)] (kg) } & 3.68(0.45) \\ 5 \text { min Apgar score (median and range) } & 9(8-10) \\ \text { Age at examination [mean (SD)] (h) } & \\ \text { Day } 1(n=47) & 12.2(4.5) \\ \text { Day 2 }(n=46) & 36.1(4.7) \\ \text { Day } 3(n=45) & 58.6(4.8)\end{array}$

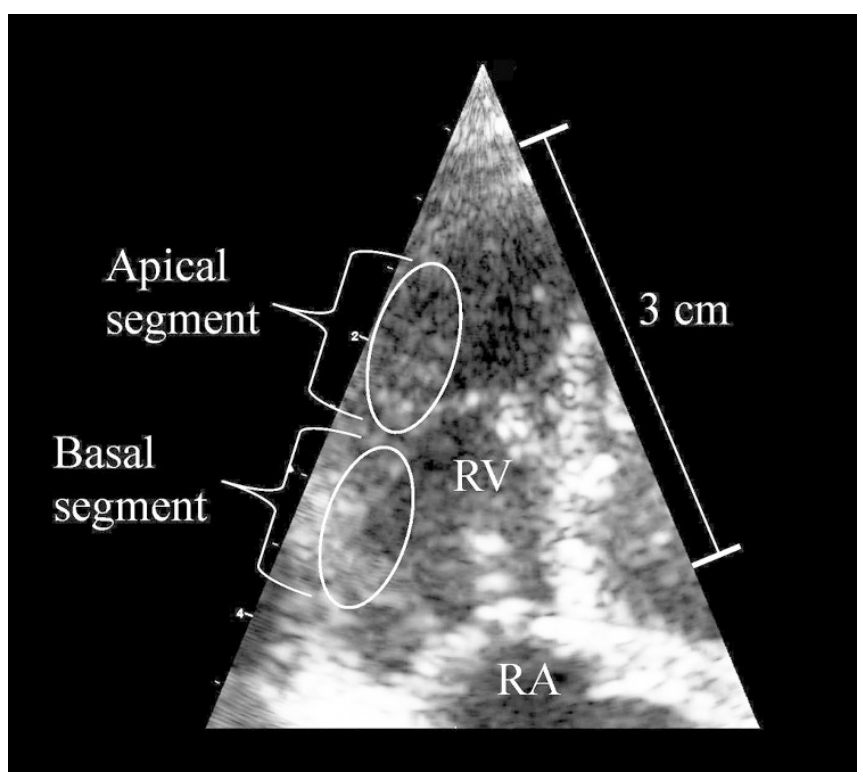

Figure 1. Grey scale image of the right superior wall from the apical superior two-chamber view, with the area from which the velocities used for the basal and apical segment analyses are collected. The scale bar shows a length of $3 \mathrm{~cm} . \mathrm{RA}=$ right atrium; RV = right ventricle.

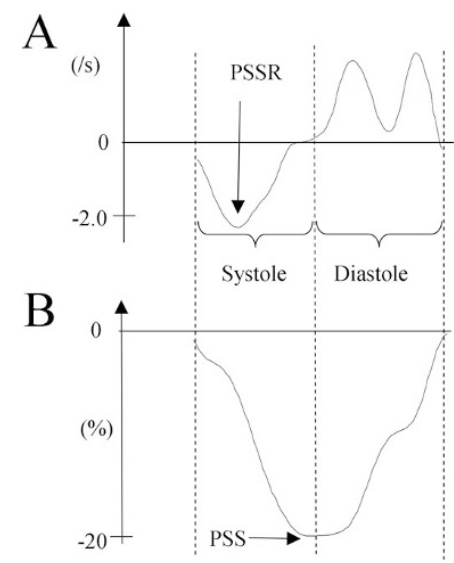

Figure 2. Example of a strain rate curve $(A)$ and a strain curve $(B)$ for one heart cycle. Arrows at the PSSR and PSS. A PSSR of $-2 / \mathrm{s}$ means that the maximum rate of shortening during systole is $2 / \mathrm{s}$. A PSS of $-20 \%$ means that the maximum shortening of the segment during systole is $20 \%$ of the segment length at start of systole.

visually for each segment. Only small deviations between the ultrasound beams and segment longitudinal length were accepted. The fractional shortening was estimated from the parasternal long axis view $(11,12)$. The ductus arteriosus flow pattern was classified in five ordinal categories (13): pulmonary hypertension pattern, growing pattern (bidirectional shunting with a predominantly left-to-right shunt), pulsatile pattern, closing pattern and closed (no flow). The ductus venosus and foramen ovale were assessed as open or closed (3). The pulmonary systolic artery pressure was estimated by applying the simplified Bernoulli equation (pressure gradient $=4 \times$ velocity $^{2}$ ) on the tricuspid valve peak regurgitation, ignoring the contribution from the right atrial blood pressure. The apical and basal left, septum, and right PSS and PSSR were estimated by averaging the values for all segments in the respective segment group eligible for analysis, and the PSS and PSSR for each examination were estimated by averaging the values for all segments in the respective examination eligible for analysis.

In normal myocardium the longitudinal PSS and PSSR are both negative, as the segment longitudinal length decreases during systole. The change in these values are here described in absolute magnitudes; i.e. a PSSR of $-1.5 / \mathrm{s}$ is described as a higher value than $-1.0 / \mathrm{s}$, and a change to the latter value is described as a decrease.

Statistics. Two sided $p$-values and 95\%CI were used. Several analyses were run in up to 18 segments, thereby carrying a high risk for Type 1 errors. Correcting the $p$-values would have led to very low significance levels and a high risk of Type 2 errors. Uncorrected $p$-values were therefore used and in the "Discussion" section, comments were made on the impact corrections would have had. Measurements between groups were compared by one-way ANOVA. Bonferroni corrections were used for post hoc pair-wise comparisons. The impacts on the measurements from the heart rate, pulmonary artery pressure, and fractional shortening were assessed by Pearson correlations and linear regression analyses. The impacts of the image quality and of the segment alignment with the ultrasound beams on the measurements were tested by multivariate linear regression analyses. In these analyses, the uneven distribution of quality and alignment assessments between segments was adjusted for. The image quality was low in the anterior left wall, and therefore only images from the other eight walls were selected for the repeatability analyses. The quality and alignment assessment were compared for two randomly selected images from each wall with Kappa scores. The PSS and PSSR were compared for the apical and basal segments from another randomly selected image from each wall with intraclass correlation coefficients and Bland-Altman plots. The segments were analyzed twice by the same investigator (E.N.) several months apart and once by another investigator (A.S.).

\section{RESULTS}

Peak systolic strain and strain rate. The absolute values for PSS and PSSR for the 18 segments analyzed are shown in Fig. 3. The PSS for each examination was $-21.8 \%(-22.1-21.4)$ (mean and 95\%CI) and the PSSR was $-1.78 / \mathrm{s}(-1.81$, $-1.74)$. Both measurements varied significantly between segments $(p<0.001)$.

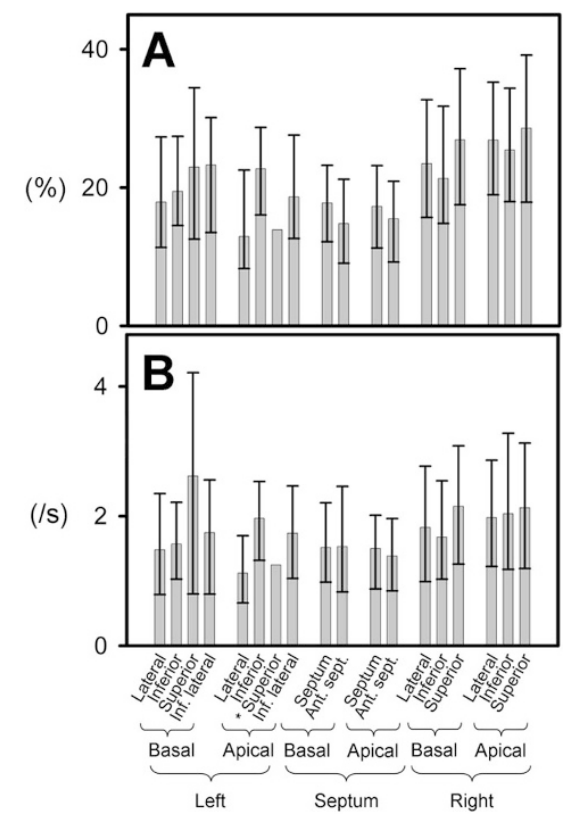

Figure 3. PSS $(A)$ and PSSR $(B)$. Absolute median with 10th and 90th percentiles. The segments are grouped by location; left, septum, and right basal and apical segments. Inf. = inferior; Ant. sept. = anterior septum. *The left superior apical segment 10th and 90th percentiles were not assessed because too few images were eligible for analyses. 
Table 2. PSS and PSSR for segments with significant variation between examinations on day 1, 2, and 3 of life

\begin{tabular}{cccc}
\hline Segment & Day 1 & Day 2 & Day 3 \\
\hline Peak systolic strain $(\%)$ & & & $-22.6(-24.1,-21.1)$ \\
Apical left inferior $(p=0.007)$ & $-20.6(-22.2,-19.0)$ & $-24(-25.7,-22.5)$ & $-21.1(-22.8,-19.5)$ \\
Basal left inferior $(p=0.042)$ & $-20.5(-22.0,-19.0)$ & $-16.3(-18.1,-14.5)$ & $-13.2(-15.0,-11.5)$ \\
Basal anterior septum $(p=0.041)$ & $-14.5(-16.1,-12.9)$ & $-26.0(-27.9,-24.1)$ & $-24.4(-26.8,-22.0)$ \\
Basal right lateral $(p=0.023)$ & $-22.0(-23.9,-20.1)$ & $-26.2(-28.4,-24.0)$ & $-29.9(-32.2,-27.5)$ \\
Basal right superior $(p=0.015)$ & $-25.3(-27.8,-22.8)$ & $-1.41(-1.55,-1.28)$ & $-1.62(-1.76,-1.49)$ \\
Peak systolic strain rate $(/ \mathrm{s})$ & $-1.68(-1.84,-1.51)$ & $-2.26(-2.49,-2.03)$ & $-2.33(-2.57,-2.09)$ \\
Basal left inferior $(p=0.025)$ & $-1.85(-2.01,-1.62)$ & & \\
Basal right superior $(p=0.010)$ &
\end{tabular}

Mean $(95 \% \mathrm{CI})$ for each days of life. $p$-value for difference between day 1, 2, and 3 (one way ANOVA).

The PSS varied significantly for the days of examination in five heart segments: basal left inferior, apical left inferior, basal anterior septum, basal right lateral, and basal right superior $(p<0.05)$ (Table 2$)$. The PSSR varied significantly in two segments: the basal left inferior and the basal right superior $(p<0.05)$.

Between genders, the PSS was different in the apical left inferiolateral segments [male $-17.2 \%(-19.4,-15.0)$, female $-20.7 \%(-22.5,-18.9), p=0.013]$ and the PSSR was different in the apical left inferiolateral segments (male $-1.91 / \mathrm{s}(-2.15$, $-1.67)$, female $-1.60 / \mathrm{s}(-1.77,-1.44), p=0.036)$.

Fetal shunts. The foramen ovale was closed in 12 examinations and open in 125 examinations, while in one examination images for assessment of foramen ovale patency was not obtained. The PSS was different between examinations with an open and a closed foramen ovale in the basal anterior septum [closed $-9.8 \%(-18.9,-0.8)$, open $-14.8 \%(-15.8$, $-13.9), p=0.029]$. The PSSR was similar for all segments. The ductus arteriosus flow showed a pulmonary hypertension pattern in 4 , a growing pattern in 22 , a pulsatile pattern in 15 , a closing pattern in 18 examinations, while in 79 examinations the duct was closed. The apical left inferior and basal anterior septum PSSR were significantly different for measurements grouped by the ductus arteriosus flow pattern (Table 3) whereas there were no differences for the PSS in any segments. Both parameters were different for examinations with an open and a closed ductus venosus in the basal right superior segments [PSSR open $-2.09 / \mathrm{s}(-2.22,-1.96)$, closed $-3.18 / \mathrm{s}(-4.36,-2.00), p<0.001$, PSS open $-26.7 \%$ $(-28.0,-25.3)$, closed $-36.4 \%(-44.4,-28.3), p=0.001]$.

Heart rate, fractional shortening, and pulmonary systolic artery pressure. The heart rate was $121 / \mathrm{min}$ (14) [mean (SD)] and had a significant impact on the apical left lateral PSSR

Table 3. PSSR values in segments with significant differences between groups based on the ductus flow pattern

\begin{tabular}{lcc}
\hline & \multicolumn{2}{c}{ Segment } \\
\cline { 2 - 3 } $\begin{array}{c}\text { Peak systolic strain rate }(/ \mathrm{s}) \\
\text { Flow pattern }\end{array}$ & $\begin{array}{c}\text { Apical left inferior } \\
(p=0.019)\end{array}$ & $\begin{array}{c}\text { Basal anterior septum } \\
(p=0.001)\end{array}$ \\
\hline Bidirectional flow & $-1.79(-2.98,-0.61)$ & $-1.33(-2.22,-0.44)$ \\
Growing flow pattern & $-1.96(-2.44,-1.48)$ & $-1.18(-1.36,-0.99)$ \\
Pulsatile & $-2.23(-2.96,-1.50)$ & $-2.53(-3.44,-1.61)$ \\
Closing pattern & $-2.42(-3.43,-1.41)$ & $-1.37(-1.79,-0.95)$ \\
No flow & $-1.91(-2.04,-1.78)$ & $-1.52(-1.69,-1.34)$ \\
\hline
\end{tabular}

Mean $(95 \% \mathrm{CI})$ in each group. $p$-value for difference between groups (one way ANOVA).
$(B=-0.010, \mathrm{SE}=0.004, p=0.018)(B=$ regression factor, $\mathrm{SE}=$ regression factor $\mathrm{SE}, p=p$-value). There was no significant impact on the PSS in any segments. The fractional shortening was $29.0 \%$ (5.7) [mean (SD)], with no differences between measurements on day 1,2 , and 3 . The correlation between the fractional shortening and the left and septum segments values were significant only for the basal left inferior PSSR ( $p=0.039$, correlation coefficient $=0.183)$. Pulmonary hypertension was not present at any examination, with tricuspid regurgitation velocities less than $3.5 \mathrm{~m} / \mathrm{s}$ in all. There were no correlations between the pulmonary systolic artery pressure and PSS or PSSR in any of the right heart segments.

Image quality and segment longitudinal alignment with the ultrasound beams. Seventy-two percent of the segments recorded could be analyzed. The fraction of images eligible for analysis varied substantially between segments (Fig. 4) and was lower in the left lateral segments than in the septum and right heart segments. The image quality had a significant impact on both the PSS $(B=1.85, \mathrm{SE}=0.216, p<0.001)$ and the PSSR $(B=0.068, \mathrm{SE}=0.025, p=0.005)$. The segment longitudinal alignment with the ultrasound beams had no impact on any measurements.

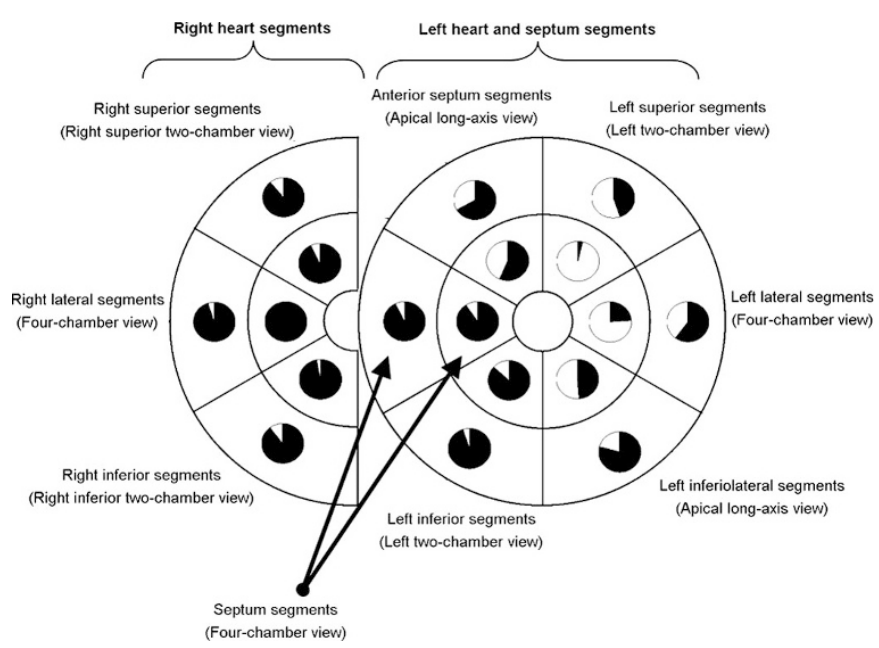

Figure 4. Pie charts of the fraction of images from each segment eligible for analysis. Each pie represents one segment. The black area of each pie shows the fraction of images possible to analyze. The heart is shown in a bull's eye projection, with left ventricle and septum segment to the right (whole circle) and right ventricle segments to the left (half circle). The pies in the inner circle show the apical segments and the pies in the outer circle show the basal segments. The name for each segment and the apical view used for recording the segment are shown. 
Left, septum and right heart apical, and basal segments. Both parameters were higher in the apical than in the basal segments in the right heart walls $(p<0.008)$ (Fig. 5) whereas there were no differences in the septum or the left heart walls. The PSS and PSSR both in the apical and in the basal segments were significantly different between the left heart, septum and right heart walls $(p<0.002)$, except for no difference between the basal left and right wall PSSR.

Repeatability analyses. The Kappa scores of the quality and alignment assessment ranged 0.53-0.60 (Table 4), and the PSS and PSSR analyses intraclass correlation coefficients ranged 0.67-0.89 (Table 4). The Bland-Altman plots for the PSS and PSSR analyses are shown in Fig. 6.

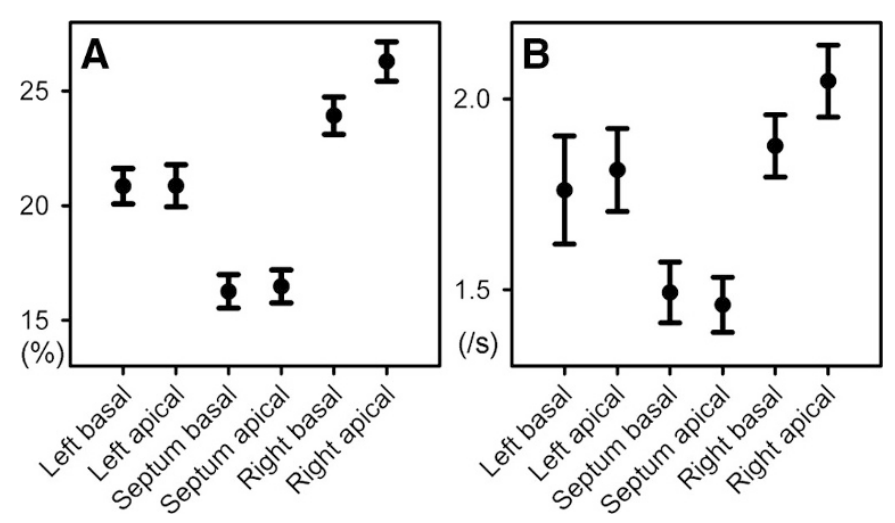

Figure 5. PSS $(A)$ and PSSR $(B)$. Absolute values (mean and $95 \% \mathrm{CI}$ ) for the basal and apical left, septum, and right heart segment groups.

Table 4. Repeatability analyses

\begin{tabular}{lccc}
\hline & $\begin{array}{c}\text { No of } \\
\text { segments }\end{array}$ & $\begin{array}{c}\text { Intra } \\
\text { observer }\end{array}$ & $\begin{array}{c}\text { Inter } \\
\text { observer }\end{array}$ \\
\hline Kappa quality & 32 & 0.58 & 0.53 \\
Kappa alignment & 32 & 0.60 & 0.57 \\
Strain ICCC & 16 & 0.89 & 0.81 \\
Strain rate ICCC & 16 & 0.85 & 0.67 \\
\hline
\end{tabular}

Kappa scores for the assessment of image quality (category 1, 2, 3, and 4; $1=$ good, $4=$ inferior for analysis) and segment/ultrasound beam alignment ( $1-3 ; 1=$ good, $3=$ inferior for analysis). Strain and strain rate ICCC. ICCC $=$ intra class correlation coefficients.

\section{DISCUSSION}

Peak systolic strain and strain rate. PSS have been found lower in fetuses (15) (left, septum and right heart values from -19 to $-17 \%$ ), higher in children aged 4-16 y (6) (left/ septum $-26 \%$, right $-32 \%$ ) and lower in adults (16) (left/ septum $-19 \%)$ than in this study $(-21.8 \%)$. As natural strain, as opposed to Lagrangian strain, were reported in children, the differences toward children are probably even larger. The increase in PSS values with increased age from neonates to childern, followed by a decrease in PSS values from children to adults, is noticeable. It has been suggested that the PSSR increases with increased age (6). PSSR have been found higher in fetuses (15) (left, septum and right $-2.1 / \mathrm{s}$ ), children (6) (left/septum $-2.0 / \mathrm{s}$, right $-2.1 / \mathrm{s})$, and lower in adults (16) (left/septum $-1.27 / \mathrm{s})$ than in this study $(-1.78 / \mathrm{s})$. Again, the differences between neonates, children, and adults are noticeable. The higher values in children might be caused by the heart growth not keeping up with increased body size; to meet the heightened cardiac demand the contractility could during growth be raised by end diastole myocardial expansion.

There were large differences between the 10th and 90th percentile values in all segments. This might reflect intrinsic variation between neonates but might also in part be caused by analysis errors due to noise.

Neither the differences between genders nor the differences between measurements on day 1,2 , and 3 showed any clear patterns, and no differences were present when the significance level were adjusted for multiple segments being analyzed.

Fetal shunts, heart rate, fractional shortening, and pulmonary systolic artery pressure. The differences regarding the fetal shunts, heart rate, fractional shortening, and pulmonary systolic artery pressure affected few segments and showed no clear patterns between fetal shunts categories, between PSS and PSSR measurements in each segment, or between adjacent segments. When adjusting the significance level for analyses being performed in multiple segments, only the differences for the ductus venosus flow in the basal right superior segments and for the ductus arteriosus flow in the basal anterior septum segments remained significant.
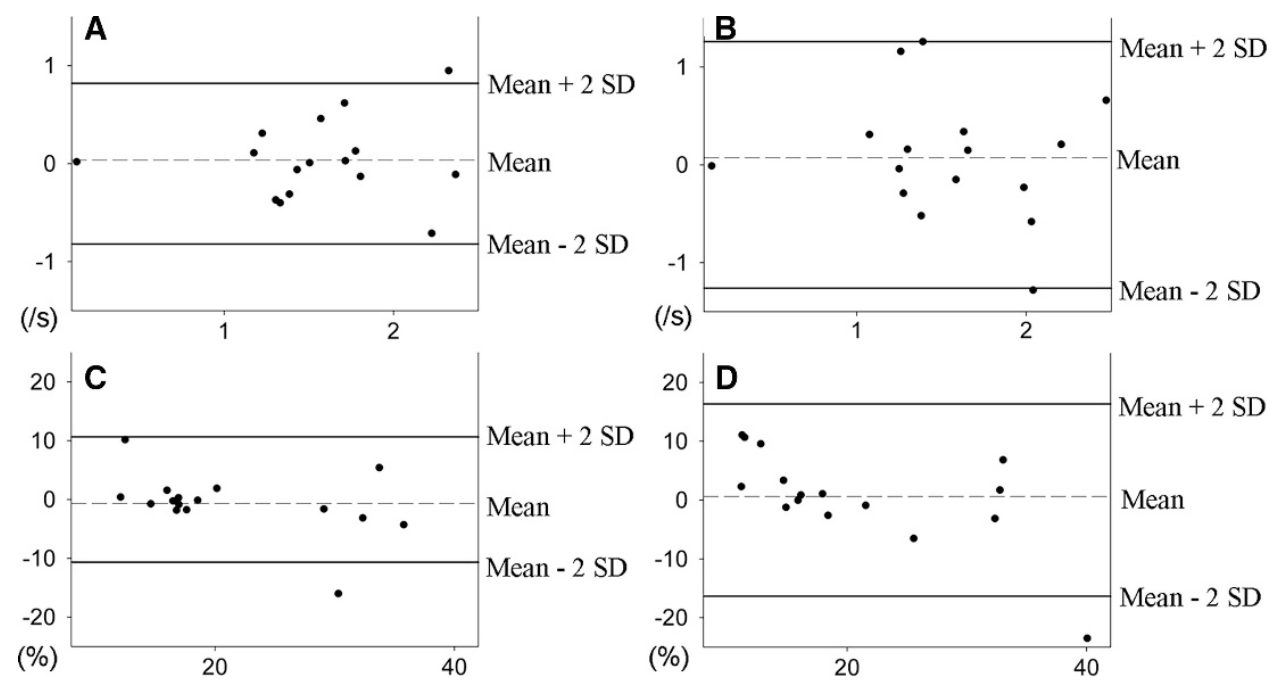

Figure 6. Repeatability analyzes (BlandAltman plots). Strain rate intra observer $(A)$, strain rate inter observer $(B)$, strain intra observer $(C)$ and strain inter observer $(D)$ measurements. $X$-axis: absolute average for the two measurements for each segment. $Y$-axis: difference between the two measurements for each segment. Dashed line: mean difference for all measurements. Solid lines: mean difference $\pm 2 \mathrm{SD}$ for the difference between the two measurements. 
The little impact on the measurements might indicate that the measurements were relatively independent of flow and volume load in this study. We therefore suggest that strain and strain rate might be used for myocardial function evaluation in neonates, relatively independent of flow and volume load. However, none of the neonates had a situation of pathologic increased right or left ventricular after load. A larger study, powered to detect smaller impacts from these factors on the measurements, must be performed before firm conclusions can be made. Further, to assess the impact from fetal shunts and pulmonary artery pressure on the measurements in neonates with circulatory disturbances, measurements must be performed in neonates with significant impact from these factors on the circulation.

In consistence with our study, the PSSR was independent of heart rate in adults in one study (17) whereas in another study (14) correlations between heart rate and PSSR in the left lateral and left posterior (inferiolateral in this study) segments at rest was found. In the latter study, there was a weak correlation between PSS and heart rate. In children (6) there was a weak correlation between heart rate and PSSR and no correlation between heart rate and PSS. The tissue Doppler derived PSS estimation involves time-integration of strain rate values. Therefore, if the PSS is independent of heart rate the average strain rate in systole must be higher at increasing heart rate. However, this increase does not necessarily apply to the peak systolic value.

Image quality and segment longitudinal alignment with the ultrasound beams. Eighty-eight percent of segments in one study in adults with infarcted hearts (16) and ninety-five percent of segments in children (6) were eligible for analysis. We found $72 \%$ of the segments in neonates eligible for analysis, and $83 \%$ from the segments examined in children. These differences might be due to differences in image quality between adults, children, and neonates, but might also reflect a higher image quality demand in neonates due to the smaller segment size.

The PSS were more prone to disturbances from noise than the PSSR. Measured by the regression analyses the difference between high and low quality images were $17 \%$ of the mean PSS and $8 \%$ of the mean PSSR. The impact from noise could be divided into two components: impact from dropouts and impact from random variation. Dropouts tend to lower both parameters, whereas the random variation could influence the PSS and the PSSR differently. The random variation will in some frames increase and in other frames decrease the measured strain rate, causing local peaks in the strain rate curve. At the point where the PSSR is measured, the random noise will often have caused a local increase in the curve and hence an increase in the measured value. The decrease in PSSR caused by dropouts will therefore often be countered by the random variation effect. Strain is estimated by integration of strain rate values, and this integration will tend to even out the random variation effect. PSS measurements will therefore be less influenced by the random variation, probably explaining the larger decrease in PSS than in PSSR measurements in noisy images.

The measured values were dependent on the image quality whereas the true PSS and PSSR values are probably not affected by the factors determining the image quality. Assessing the quality is therefore clinically relevant and important. Poor-quality images should be excluded from analysis. Ob- taining objective criteria for image quality assessment will probably improve the method's feasibility, and before such criteria are established care must be taken when comparing measurements between studies.

The segment longitudinal alignment with the ultrasound beams had no impact on the measured values. Similar findings have been shown for strain measurements in adults (18). Images with poor alignment should not be analyzed, as the measured strain rate could be reduced to zero at a $30^{\circ}$ angle (Heimdal A. Doppler Based Ultrasound Imaging Methods for Noninvasive Assessment of Tissue Viability (Thesis). Trondheim, Norway: Norwegian University of Science and Technology). We speculate that differences might have been found in our study if poorer alignment between the ultrasound beams and segment longitudinal length had been accepted.

Left, septum and right heart apical, and basal segments. Differences between left and right heart measurements might be caused by different dispersion of longitudinal and circular myocardial fibers (7). In agreement with this, values have been found higher in the right than in the left heart in this study, in children (6) and in adults (14). The higher left heart than septum values in neonates is consistent with findings in adults in one study (14) but not in another study (17). In accordance with our results, annulus velocities have been found highest in the right wall, intermediate in the left wall, and lowest in the septum in adults (19) and in neonates (20).

As in this study, right heart measurements in adults increase toward the apex (14). We found no differences between apical and basal segment values in the septum or in the left heart. This is consistent with findings in one study in adults (14), whereas higher basal than mid and apical values were found in adults in another study (17).

Clinical use. As there were little or no impacts from gender, age, normal fetal shunts, pulmonary systolic artery pressure, and heart rate on the measurements, strain and strain rate reflect myocardial function in neonates. It is not known how the measurements are influenced in pathologic states. Images eligible for analyses were obtained more often from the inferior left heart, septum and right heart, while few images eligible for analyses were obtained from the lateral and superior left ventricle. Standardized methods for evaluation of the right heart function are not well established and in many clinical situations in neonates, the right heart function is of major interest. Therefore, tissue Doppler imaging might be a tool more suitable for myocardial function evaluation in the right heart than in the left heart.

There were large differences between the measurements in each segment and there were also significant differences in the repeatability analyses. Strain and strain rate in neonates might have the potential for quantifying differences between segment groups and patient groups. Further refinement of the method might be required before differences between individuals and between segments within individuals can be assessed.

Limitations. The measurements presented here were assessed in neonates within a narrow range for age and normal birth weight and were recorded during the time where the major postpartum changes from fetal via transitional to infant circulation occurs. It is not known how these measurements relate to measurements later in the neonatal period. For some 
segments, only a few images could be analyzed. The relative load independency of strain and strain rate in this study should be confirmed in larger studies.

Future studies. Strain and strain rate should be assessed in neonatal patient groups with affected heart function and circulation. Strain and strain rate analysis using more simplified procedures, e.g. analyze one segment per wall or performing analyzes with the segment set stationary in space (as opposed to using a tracking algorithm to keep the segment within the same myocardial region throughout the cardiac cycle) should be investigated. Methods for objective assessment of the image quality should be explored.

In conclusion, PSS and PSSR in neonates aged 1-3 $\mathrm{d}$ are presented. The measured variation was high within segments, between segments and between individuals. At present, the method might be feasible for assessing differences between segment groups and patient groups. Age, gender, fetal shunts, heart rate, and pulmonary systolic artery pressure had little impact on the measurements, indicating that under normal circumstances the methods might be used to evaluate myocardial function regardless these factors. The measurements were affected by image quality, and poor-quality images should be excluded. Images eligible for analyses were obtained more often in the inferior left heart, the septum and the right heart. Values were highest in the right wall, intermediate in the left wall and lowest in the septum. Values were higher in the apical than in the basal segments in the right heart walls and not in the septum or left heart walls.

Acknowledgments. We thank Leif Sandvik, Centre for Clinical Research, Ullevål University Hospital, for advise on the statistical analyses. We thank Rolf Lindemann, NICU, Ullevål University Hospital for valuable comments during the preparation of the final draft.

\section{REFERENCES}

1. Heimdal A, Stoylen A, Torp H, Skjaerpe T 1998 Real-time strain rate imaging of the left ventricle by ultrasound. J Am Soc Echocardiogr 11:1013-1019

2. Nestaas E, Stoylen A, Sandvik L, Brunvand L, Fugelseth D 2007 Feasibility and reliability of strain and strain rate measurement in neonates by optimizing the analysis parameters settings. Ultrasound Med Biol 33:270-278

3. Fugelseth D, Lindemann R, Liestol K, Kiserud T, Langslet A 1997 Ultrasonographic study of ductus venosus in healthy neonates. Arch Dis Child Fetal Neonatal Ed 77:F131-F134
4. Nestaas E, Stoylen A, Fugelseth D 2008 Optimal types of probe, and tissue Doppler frame rates, for use during tissue Doppler recording and off-line analysis of strain and strain rate in neonates at term. Cardiol Young 18:502-511

5. Cerqueira MD, Weissman NJ, Dilsizian V, Jacobs AK, Kaul S, Laskey WK, Pennell DJ, Rumberger JA, Ryan T, Verani MS 2002 Standardized myocardial segmentation and nomenclature for tomographic imaging of the heart: a statement for healthcare professionals from the Cardiac Imaging Committee of the Council on Clinical Cardiology of the American Heart Association. Circulation 105:539-542

6. Weidemann F, Eyskens B, Jamal F, Mertens L, Kowalski M, D'hooge J, Bijnens B, Gewillig M, Rademakers F, Hatle L, Sutherland GR 2002 Quantification of regional left and right ventricular radial and longitudinal function in healthy children using ultrasound-based strain rate and strain imaging. J Am Soc Echocardiogr 15:20-28

7. Naito H, Arisawa J, Harada K, Yamagami H, Kozuka T, Tamura S 1995 Assessment of right ventricular regional contraction and comparison with the left ventricle in normal humans: a cine magnetic resonance study with presaturation myocardial tagging. Br Heart J 74:186-191

8. Klein SS, Graham TP Jr., Lorenz CH 1998 Noninvasive delineation of normal right ventricular contractile motion with magnetic resonance imaging myocardial tagging. Ann Biomed Eng 26:756-763

9. D'hooge J, Heimdal A, Jamal F, Kukulski T, Bijnens B, Rademakers F, Hatle L, Suetens P, Sutherland GR 2000 Regional strain and strain rate measurements by cardiac ultrasound: principles, implementation and limitations. Eur J Echocardiogr $1: 154-170$

10. Aase SA, Stoylen A, Ingul CB, Frigstad S, Torp H 2006 Automatic timing of aortic valve closure in apical tissue Doppler images. Ultrasound Med Biol 32:19-27

11. Lai WW, Geva T, Shirali GS, Frommelt PC, Humes RA, Brook MM, Pignatelli RH, Rychik J 2006 Guidelines and standards for performance of a pediatric echocardiogram. J Am Soc Echocardiogr 19:1413-1430

12. Lang RM, Bierig M, Devereux RB, Flachskampf FA, Foster E, Pellikka PA, Picard MH, Roman MJ, Seward J, Shanewise JS, Solomon SD, Spencer KT, Sutton MS, Stewart WJ 2005 Recommendations for chamber quantification: a report from the American Society of Echocardiography's Guidelines and Standards Committee and the Chamber Quantification Writing Group, developed in conjunction with the European Association of Echocardiography, a branch of the European Society of Cardiology. J Am Soc Echocardiogr 18:1440-1463

13. Su BH, Watanabe T, Shimizu M, Yanagisawa M 1997 Echocardiographic assessment of patent ductus arteriosus shunt flow pattern in premature infants. Arch Dis Child Fetal Neonatal Ed 77:F36-F40

14. Kowalski M, Kukulski T, Jamal F, D'hooge J, Weidemann F, Rademakers F, Bijnens B, Hatle L, Sutherland GR 2001 Can natural strain and strain rate quantify regional myocardial deformation? A study in healthy subjects. Ultrasound Med Biol 27:1087-1097

15. Di Salvo G, Russo MG, Paladini D, Pacileo G, Felicetti M, Ricci C, Cardaropoli D, Palma M, Caso P, Calabro R 2005 Quantification of regional left and right ventricular longitudinal function in 75 normal fetuses using ultrasound-based strain rate and strain imaging. Ultrasound Med Biol 31:1159-1162

16. Voigt JU, Arnold MF, Karlsson M, Hubbert L, Kukulski T, Hatle L, Sutherland GR 2000 Assessment of regional longitudinal myocardial strain rate derived from doppler myocardial imaging indexes in normal and infarcted myocardium. J Am Soc Echocardiogr 13:588-598

17. Andersen NH, Poulsen SH 2003 Evaluation of the longitudinal contraction of the left ventricle in normal subjects by doppler tissue tracking and strain rate. J Am Soc Echocardiogr 16:716-723

18. Storaa C, Aberg P, Lind B, Brodin LA 2003 Effect of angular error on tissue Doppler velocities and strain. Echocardiography 20:581-587

19. Alam M, Wardell J, Andersson E, Samad BA, Nordlander R 1999 Characteristics of mitral and tricuspid annular velocities determined by pulsed wave Doppler tissue imaging in healthy subjects. J Am Soc Echocardiogr 12:618-628

20. Mori K, Nakagawa R, Nii M, Edagawa T, Takehara Y, Inoue M, Kuroda Y 2004 Pulsed wave Doppler tissue echocardiography assessment of the long axis function of the right and left ventricles during the early neonatal period. Heart 90:175-180 